\title{
Two Mechanisms for Task Switching in the Prefrontal Cortex
}

\author{
Alexandre Hyafil, ${ }^{1,2 \star}$ Christopher Summerfield, ${ }^{4 \star}$ and Etienne Koechlin ${ }^{1,2,3}$ \\ ${ }^{1}$ Institut National de la Santé et de la Recherche Médicale, ${ }^{2}$ Ecole Normale Supérieure, and ${ }^{3}$ Center for NeuroImaging Research, University Pierre and Marie \\ Curie (Paris 6), Groupe Hospitalier Pitié-Salpêtrière, 75005 Paris, France, and ${ }^{4}$ Department of Experimental Psychology, Oxford University, Oxford 0X1 \\ 3UD, United Kingdom
}

\begin{abstract}
Measuring the cognitive and neural sequelae of switching between tasks permits a window into the flexible functioning of the executive control system. Prolonged reaction times (RTs) after task switches are accompanied by increases in brain activity in the anterior cingulate cortex (ACC) and dorsolateral prefrontal cortex (dlPFC), but the contribution made by these regions to task level control remains controversial. Here, subjects performed a hybrid spatial Stroop/task-switching paradigm, requiring them to respond with a joystick movement to congruent or incongruent spatial/verbal cues. Relative to the previous trial, the active task either switched or remained the same. Calculating switch costs as a function of current and previous trial congruency, we observed both a general RT increase on every switch trial, and additional slowing and impairment to performance when the switch occurred on the second of two successive incongruent trials ( $i$ I trials). Imaging data revealed corresponding neural concomitants of these two switch costs: the ACC was activated by task switches regardless of trial type (including congruent trials in which task-relevant and task-irrelevant information did not clash), whereas the caudal dlPFC exhibited a switch cost that was unique to $i$ I trials. We argue that the ACC configures the priorities associated with a new task, whereas the dlPFC tackles interference from recently active, rivalrous task sets. These data contribute to a literature arguing that human cognitive flexibility benefits from the setting of new priorities for future action as well as the overcoming of interference from previously active task sets.
\end{abstract}

\section{Introduction}

Humans and other primates can shift flexibly between competing tasks to meet the changing needs of the environment. However, doing so engenders a prolongation of reaction times (RTs) at the transition between tasks (Jersild, 1927; Monsell, 2003). Despite ongoing controversy, this "switch cost" seems to be attributable to multiple neurocognitive factors, including the overcoming of "proactive" interference from the previous task set (Allport, 1994; Yeung et al., 2006) and the engagement of time-consuming control processes that allow the selection and/or deselection of context-appropriate stimulus-response contingencies ("task set") (Rogers and Monsell, 1995; Monsell, 2003). These control processes are among the fundamental building blocks of higher cognitive performance, and a key goal of contemporary neuroscience is to understand their neural basis.

A diverse brain network becomes active during task switching, yielding a number of good candidates for controlling task engagement and disengagement. Among the most prominent of these are the anterior cingulate cortex (ACC) and the dorsolateral prefrontal cortex (dlPFC), whose respective contributions to executive control are still vigorously debated. Many theorists hold that

\footnotetext{
Received June 19, 2008; revised Dec. 5, 2008; accepted Dec. 11, 2008.

This work was supported by a European Young Investigator Award (E.K.) and a prize from The BettencourtSchueller Foundation. We thank Thomas Jubault, Eric Bertasi, and Kévin Nigaud for technical assistance, and Tobias Egner for helpful comments on a previous version of this manuscript.

${ }^{*}$ A.H. and C.S. contributed equally to this work.

Correspondence should be addressed to Etienne Koechlin, Ecole Normale Supérieure, 29, rue d'Ulm, 75005 Paris, France. E-mail: etienne.koechlin@upmc.fr.

DOI:10.1523/JNEUROSCI.2828-08.2009

Copyright $\odot 2009$ Society for Neuroscience $\quad$ 0270-6474/09/295135-08\$15.00/0
}

the ACC adjusts performance according to task demands or likely rewards (Carter et al., 1998; Botvinick et al., 2001; Rushworth et al., 2004): for example, ACC responses track the increased efficiency of task processing observed after a trial that provoked response conflict (Gratton et al., 1992; Kerns et al., 2004). In contrast, the dIPFC is required to "fend off" information that might otherwise interfere with action selection (e.g., resolving conflict, or protecting tonically maintained information from disruption). It is this functional dissociation between readying the cognitive system for an expected task, and tackling interference from competing processes, that inspired our hypothesis that ACC and dIPFC contribute, respectively, to the configuration of new task priorities, and overcoming of competition from a rival task set, during executive performance.

We tested this hypothesis using a task that combines elements from two classic probes of executive function: the spatial Stroop and task-switching paradigms (Goschke, 2000; Aron et al., 2004; Brown et al., 2007). Subjects undergoing functional brain imaging viewed concurrent spatial/verbal cues that prompted either common or conflicting joystick movements (Fig. 1). The active task (spatial or verbal) could either remain the same, or switch, from trial to trial. This design allowed us to dissociate demands of configuring the priorities associated with a new task set regardless of current demand (i.e., on every switch trial, independent of the congruence between spatial and verbal cues) from those of resolving competition from the previously implemented task set [on incongruent switch trials, notably those preceded by another incongruent trial (switch-iI trials)]. Brain imaging data revealed that the ACC exhibited higher activity on every switch trial, whereas switch costs in the dlPFC were confined to iI trials, sug- 

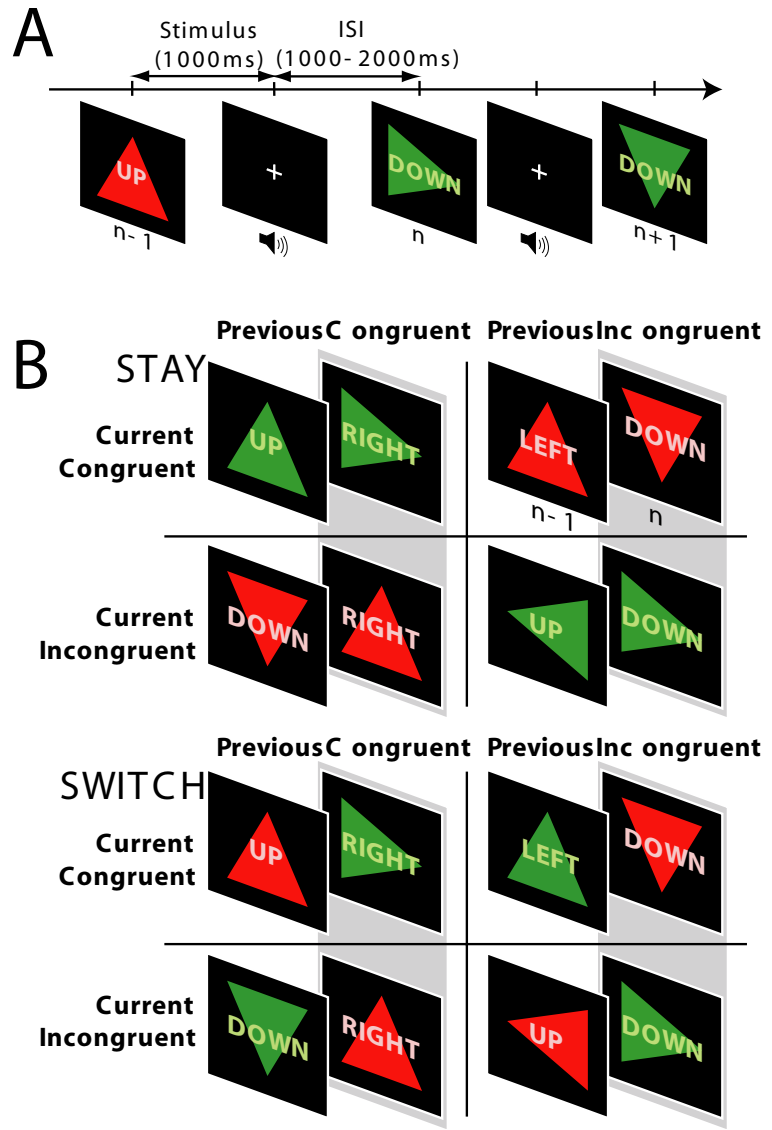

Figure 1. Behavioral paradigm. $A$, Example stimulus sequence. Stimuli consisted of a red or green triangle/word ensemble presented for $1000 \mathrm{~ms}$ and followed by an interstimulus interval of $500-1500 \mathrm{~ms}$, in which a lone central fixation occupied the screen. A brief auditory feedback tone at stimulus offset signaled whether the response was correct or incorrect. $\boldsymbol{B}$, Trial classification by current and previous congruence for stay (top) and switch (bottom) trials. Example trials appear within the gray-shaded boundary, preceded by a congruent trial (left) or incongruent trial (right). For example, the top/top right trial marked " $n$ " is a congruent trial (the word "down" on a downward-pointing triangle) preceded by an incongruent trial (the word "left" on an upward-pointing triangle, marked " $n-1$ "). The trial is a stay trial because the color of the ensemble repeats; this trial is thus classified as a stay-ic trial. Together, the top four quadrants for stay and the bottom four quadrants for switch make up the eight cells in our $2 \times 2 \times 2$ factorial design.

gesting that these two structures make dissociable contributions to task switching.

\section{Materials and Methods}

Subjects. Twenty-four right-handed neurologically normal individuals between the ages of 18 and 30 participated in the experiment. Subjects all had normal or corrected-to-normal vision and were recruited on campus at the Université Pierre et Marie Curie in Paris, France. Subjects all gave informed consent during an interview with our on-site physician and were paid 120 euros for their participation.

Stimuli and procedure. All stimuli were generated and presented using PsychToolBox (Brainard, 1997) and appeared on a uniform black background. The experiment consisted of six experimental runs of 128 trials $(\sim 7 \mathrm{~min})$ each. The paradigm was a variant of the spatial Stroop in which the relevant task set (spatial or verbal) could either switch, or stay the same, from trial to trial. On each trial, a stimulus appeared centrally for 1000 ms, composed of one of the French words "haut," "bas," "gauche," and "droite" (meaning "up," "down," "left," and "right," respectively) superimposed on a slightly darker isosceles triangle, which could point leftward, rightward, up, or down. On these trials, one-half of the stimuli were congruent [e.g., the word "gauche" (left) on a triangle pointing left], and one-half were incongruent [e.g., the word "haut" (up) together with a triangle pointing right]; these were each followed with equal probability by congruent or incongruent trials. Each of the instances of task (spatial, verbal), congruence (congruent, incongruent), and response (left, right, up, down) was equally frequent, and transitions between all of these classes of trial were equally probable.

Subjects were instructed to respond to these stimuli by moving a joystick with their right hand toward one of the four cardinal directions. Responses were detected when the joystick was displaced to threshold within $45^{\circ}$ of any of the four directions. The color of the stimulus ensemble, which could be red (50\% trials) or green (50\% trials), indicated whether the word or triangle was task relevant on any given trial (colortask associations were counterbalanced between subjects). A $300 \mathrm{~ms}$ auditory feedback was given at the end of the $1000 \mathrm{~ms}$ presentation period: a high tone $(880 \mathrm{~Hz})$ rewarded correct responses, whereas errors [incorrect responses and time-outs (i.e., failures to respond within the $1 \mathrm{~s}$ time window)] were indicated by a low tone $(440 \mathrm{~Hz})$. One notable effect of this $1 \mathrm{~s}$ deadline was the transfer of a proportion of the behavioral effects from the reaction time data to the error rate data: late responses were counted as errors. The duration of the interval interposed between stimuli was randomly drawn from a normal distribution (mean, $1.5 \mathrm{~s}$; range, $1-2 \mathrm{~s}$ ). Each run began with $10 \mathrm{~s}$ lead in and ended with $\sim 20 \mathrm{~s}$ lead out in which only a fixation cross was visible. All subjects had been trained on this task on an equivalent 30 min session a few days before the scan.

Functional magnetic resonance image data acquisition and preprocessing. Functional magnetic resonance images (fMRIs) were acquired with a Thermo Fisher Scientific Allegra 3.0 T scanner to acquire gradient echo $\mathrm{T} 2^{\star}$-weighted echoplanar images with blood oxygenation leveldependent contrast as an index of local increases in synaptic activity. The image parameters used were as follows: matrix size, $64 \times 64$; voxel size, $2 \times 2 \mathrm{~mm}$; echo time, $40 \mathrm{~ms}$; repetition time, $2000 \mathrm{~ms}$. A functional image volume comprised 32 contiguous slices of $3 \mathrm{~mm}$ thickness (with a $1 \mathrm{~mm}$ interslice gap), which ensured that the whole brain was within the field of view.

Data were preprocessed using SPM2 (Wellcome Department of Cognitive Neurology, London, UK). Functional images were corrected for head motion and differences in slice acquisition timing. Functional data were then spatially normalized to a template based on the Montreal Neurological Institute stereographic space using a 12-parameter affine transformation along with a nonlinear transformation using cosine basis functions. Images were resampled to $3 \mathrm{~mm}$ cubic voxels and spatially smoothed with a $10 \mathrm{~mm}$ full-width at half-maximum isotropic Gaussian kernel. A 128 s temporal high-pass filter was applied to exclude lowfrequency artifacts. Temporal correlations were estimated using restricted maximum likelihood estimates of variance components using a first-order autoregressive model. The resulting nonsphericity was used to form maximum-likelihood estimates of the activations.

Statistical analyses. We conceived of the experimental paradigm as a factorial $2 \times 2 \times 2$ design, with factors current trial congruence $(C$ or $I)$, previous trial congruence ( $c$ or $i$ ), and task set switch (switch or stay). Behavioral data (accuracy and RTs) were analyzed using factorial ANOVAs in combination with post hoc $t$ tests. The following types of trial were excluded from all behavioral analyses: the first trial of every block, trials on which either the full stimulus ensemble or the response was repeated, and trials after an error. Eliminating these trials from the analyses ensured that switch and/or conflict adaptation effects were not mere artifacts of stimulus or response priming. RT analyses were limited to correct trials. An $\alpha$ of $p<0.05$ was used for all behavioral analyses.

For brain imaging data, statistical models were generated using SPM2 under the assumptions of the general linear model. Regressors were constructed by convolving the onsets of stimuli in each of the eight conditions with the canonical hemodynamic response function. Additional nuisance regressors excluded variance associated with the first trial on each block, errors ( $\sim 7 \%$ trials), trials preceded by an error $(7 \%)$, repetitions of the full stimulus ensemble ( $\sim 6 \%$ trials), and response repetitions $(\sim 25 \%)$. Non-neural artifacts in the time series were also modeled separately with the inclusion of a regressor encoding the mean signal from 1000 voxels randomly selected from the space outside the brain. For the principal analyses, SPMs at the second (between-subjects) level were computed to identify voxels exhibiting significant ( $p<0.001$, uncor- 

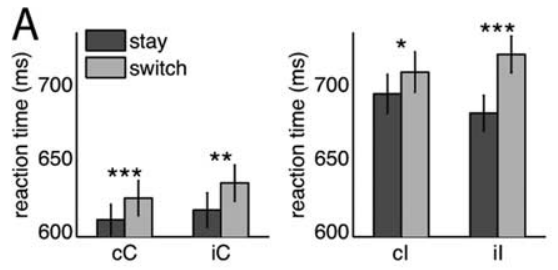

B
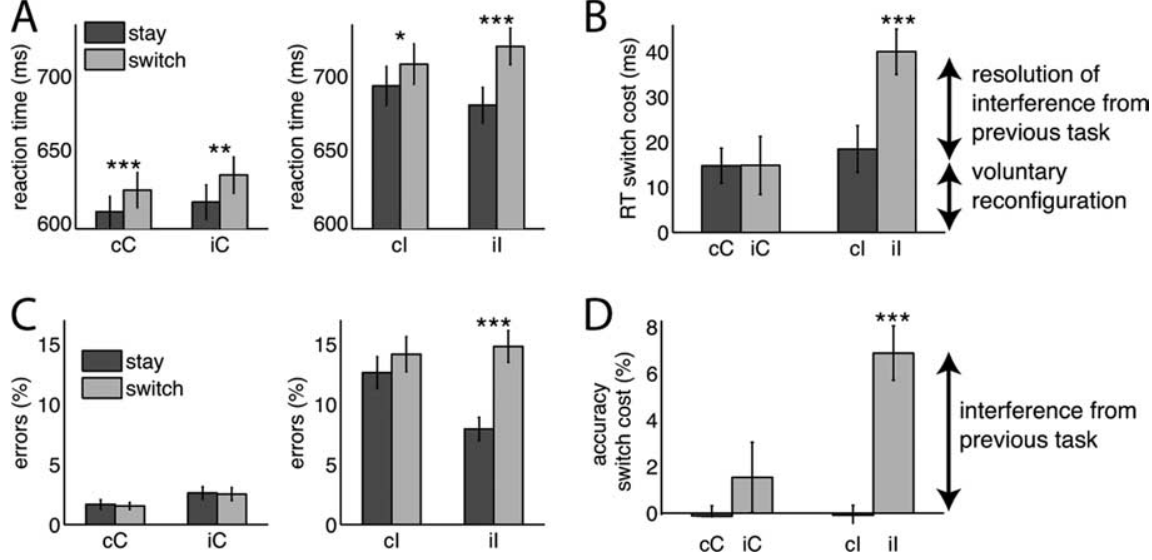

Figure 2. Behavioral switch costs. A, Mean RTs (in milliseconds) in each condition for stay (dark gray bars) and switch (light gray bars) conditions, for current congruent ( $\mathrm{C} C$ and $i C$ trials (left) and current incongruent ( $\mathrm{Cl}$ and il) trials (right). Error bars represent the SEM. B, Switch costs, defined as the mean RT difference between switch trials and stay trials, for current congruent (left), current incongruent trials (right), divided into previous congruent (dark gray bars) and previous incongruent (light gray bars). $\boldsymbol{C}$, Mean percentage error rate in the different trial conditions. The legend is as for $\boldsymbol{A}$. $\boldsymbol{D}$, Switch cost plotted as increased percentage of errors for switch trials. The legend is as for $\boldsymbol{B}$. The asterisks denote level of statistical significance $\left({ }^{*} p<0.05\right.$; ${ }^{* *} p<$ $0.01 ; * * *<0.001)$.

rected) responses on all switch trials compared with stay trials, and those responding uniquely on switch-iI trials compared with stay-iI trials. Parameter estimates obtained within regions of interest (ROIs) responding to these contrasts were subsequently extracted and factorial ANOVAs were used to confirm their sensitivity to the main effects of switch, congruence, previous congruence, and their two- and three-way interactions.

Because it is known that overlap between stimulus features in the previous and current trials can have an important impact on behavioral and neural measures of cognitive control (Mayr et al., 2003; Hommel et al., 2004; Ullsperger et al., 2005), we conducted a control analysis in which trials in which either the word feature and/or the triangle feature was repeated $(\sim 33 \%)$ were excluded. All the effects reported here were robust to the inclusion/exclusion of such "partial stimulus repetition" trials.

\section{Results}

\section{Behavioral results: task switching}

Overall, subjects were $\sim 20$ ms slower on switch than stay trials, and this effect was statistically reliable for trials comprising all combinations of previous and current congruence (Fig. 2A) (all $t$ scores, $>3.5$; $p<0.005)$. However, the effect was not uniform across trial types (Fig. $2 B$ ): iI trials evoked significantly larger switch costs $(\sim 40 \mathrm{~ms})$ than all three other trial types ( $c C$ and $c I$, $15 \mathrm{~ms} ; i C, 18 \mathrm{~ms}$ ), which did not differ from each other. Formally, there was both a main effect of switch $\left(F_{(1,23)}=27.5 ; p<1 \times\right.$ $\left.10^{-4}\right)$ and interactions between switch and congruency $\left(F_{(1,23)}=\right.$ $12.1 ; p<0.003)$, between switch and previous congruency $\left(F_{(1,23)}\right.$ $\left.=17.2 ; p<1 \times 10^{-3}\right)$, and, critically, a significant interaction between all three factors $\left(F_{(1,23)}=7.3 ; p<0.05\right)$. Accuracy varied even more dramatically with the interaction between factors: subjects were less accurate for switch-iI than stay-iI trials $(\sim 7 \%$ more errors; $\left.t_{(23)}=5.9 ; p<10^{-5}\right)$, but this difference between switch and stay was not statistically significant for any other combination of current of previous congruence (all values of $p>0.3$ ) (Fig. 2C,D). The large switch cost for $i$ trials was the main source of significant interactions between switch and current congruence $\left(F_{(1,23)}=30.8 ; p<10^{-4}\right)$, previous congruence $\left(F_{(1,23)}=\right.$ $5.7 ; p<0.05)$, as well as a three-way interaction $\left(F_{(1,23)}=5.9 ; p<\right.$ $0.05)$. Crucially, therefore, the results suggest that at least two statistically independent factors contribute to switch costs (Fig.
2). One factor (visible only in the RT data) reflects a general prolongation of processing time on switch relative to stay trials, regardless of the congruence of the current or previous stimulus (Fig. 2B). However, an additional switch cost, reflected both in increased RTs and increased likelihood of errors, is seen specifically on iI trials, in which subjects are required not only to engage a new task set but also to disengage that which was active on the previous trial (Fig. $2 B, D$ ).

Behavioral results: conflict adaptation Although subjects were slower for incongruent trials than for congruent trials $\left(F_{(1,23)}=189 ; p<10^{-11}\right)$, this effect was diminished after incongruent trials (main effect of previous congruency: $F_{(1,23)}=$ 4.6, $p<0.05$; interaction of previous and current congruencies: $F_{(1,23)}=8.1, p<$ $0.01)$. This replicates the previously described "conflict adaptation" effect (Gratton et al., 1992) whereby reaction times are decreased to the second of two trials involving conflict (iI faster than $c I$ ). However, as described above, the three-way interaction between switch, previous congruence, and current congruence indicated that beneficial conflict adaptation occurred only for stay trials $\left(F_{(1,23)}=12.1 ; p<0.003\right)$ and not for switch trials $(p>$ $0.7)$ : in fact, we observed a reversal, whereby subjects were actually slower for switch-iI trials than for switch-cI trials $\left(t_{(23)}=3.1\right.$; $p<0.005)$. Similarly, subjects also committed more errors for incongruent trials $\left(F_{(1,23)}=127 ; p<10^{-10}\right)$, and this effect was reduced overall after incongruent trials (interaction: $F_{(1,23)}=$ $13.4 ; p<0.005)$. Once again, however, this effect was driven by stay $\left(F_{(1,23)}=19.2 ; p<0.001\right)$ and not switch $(p>0.8)$ trials. Consistent with previous reports (Goschke, 2000; Monsell et al., 2003; Brown et al., 2007), thus, beneficial conflict adaptation effects in our experiment were confined to stay trials.

\section{Brain imaging results}

Comparing switch $>$ stay trials activated a well described brain network including medial and lateral portions of the frontal and parietal cortices, visual regions, and the cerebellum. A full list of activated clusters surviving a threshold of $p<0.001$, uncorrected, is reported in Table 1. However, the main object of our fMRI analyses was to identify brain regions associated with the two dissociable components of task-switching present in the behavioral data (Fig. $2 B, D$ ). We began by focusing on three small clusters observed on the medial surface of the frontal lobe, falling on the dorsal anterior cingulate gyrus (ACC; peak, 0, 6, 36), which merged into a single cluster when the threshold was lowered to $p<0.005$. This ACC region (Fig. $3 A$ ) was sensitive to the main effect of switch $\left(F_{(1,23)}=18.3 ; p<0.001\right)$ and congruence $\left(F_{(1,23)}\right.$ $=18.7 ; p<0.001)$. Although initial, visual inspection of the mean data appeared to show a trend for the ACC to respond more on switch-iI trials, neither the interaction between switch and congruence $(p>0.8)$ nor that between switch and previous congruence $(p>0.9)$ was statistically significant, nor was the threeway interaction $(p>0.4)$. Consistently, neural switch costs in the ACC did not statistically differ on $i I, i C, c I$, and $c C$ trials (Fig. 3C) (all values of $p>0.4$ ).

In contrast, when switch costs on associated with $i$ trials alone 
Table 1. Voxels responsive to switch $>$ stay, at an uncorrected threshold of $p<0.001$

\begin{tabular}{|c|c|c|c|c|c|c|}
\hline \multirow[b]{2}{*}{ Cluster size } & \multirow[b]{2}{*}{$t$ value } & \multicolumn{3}{|c|}{ MNI coordinate } & \multirow[b]{2}{*}{ Brodmann's area (BA) } & \multirow[b]{2}{*}{ Brain region } \\
\hline & & $x$ & $y$ & $z$ & & \\
\hline \multirow[t]{3}{*}{1578} & 6.05 & 18 & -42 & 0 & \multirow[t]{3}{*}{ 18/19/30/31 } & \multirow[t]{3}{*}{ Visual cortex/cerebellum } \\
\hline & 6.01 & 24 & -48 & 3 & & \\
\hline & 5.84 & -18 & -42 & 3 & & \\
\hline 52 & 5.21 & -15 & -96 & -15 & $17 / 18$ & Visual cortex \\
\hline 73 & 5.00 & 3 & -15 & 33 & $24 / 23 / 31$ & Posterior cingulate cortex \\
\hline 22 & 4.98 & -9 & -15 & 0 & & Thalamus / brainstem \\
\hline \multirow[t]{2}{*}{26} & 4.46 & 30 & 39 & 21 & \multirow[t]{2}{*}{10} & \multirow[t]{2}{*}{ Anterior PFC } \\
\hline & 3.61 & 30 & 51 & 33 & & \\
\hline 9 & 4.26 & 33 & -42 & -24 & 20 & Temporal cortex \\
\hline 13 & 4.23 & 0 & 6 & 36 & $24 / 32$ & Anterior cingulate cortex \\
\hline 10 & 4.01 & 6 & -42 & 27 & 31 & Posterior cingulate cortex \\
\hline 11 & 3.93 & 9 & 18 & 42 & 6 & Pre-SMA \\
\hline 5 & 3.90 & -27 & 36 & 36 & 9 & Anterior PFC \\
\hline 6 & 3.74 & -33 & 36 & 15 & 9 & Anterior PFC \\
\hline 8 & 3.71 & 6 & 9 & 54 & 6 & SMA \\
\hline
\end{tabular}

From left to right are the following: cluster size in voxels $(3 \times 3 \times 3 \mathrm{~mm}$ ); peakt value (with $23 \mathrm{df}) ; x, y, z$ coordinates of local maximum in the standard space of Montreal Neurological Institute (MNI) template brain; approximate Brodmann's area (BA); and label given to brain region in the text, if mentioned. PFC, Prefrontal cortex; SMA, supplementary motor area.

were assessed, a large cluster falling in the left caudal dlPFC (peak, -54, 0, 36) was observed (Fig. $3 B-D$, Table 2$)$. In addition to a main effect of congruence $\left(F_{(1,23)}=\right.$ 41.7; $\left.p<10^{-5}\right)$, the caudal dlPFC displayed a main effect of switch $\left(F_{(1,23)}=\right.$ $4.71 ; p<0.05)$ and interaction between switch and previous congruence $\left(F_{(1,23)}=\right.$ 8.04; $p<0.009$ ), both of which were driven by an interaction between all three factors $\left(F_{(1,23)}=6.08 ; p<0.03\right)$. Moreover, unlike in the ACC, in the caudal dlPFC the neural switch cost on iI trials was significantly larger than on $c C\left(t_{(23)}=\right.$ $3.3 ; p<0.004), c I\left(t_{(23)}=3.6 ; p<0.002\right)$, and $i C\left(t_{(23)}=2.8 ; p<0.02\right)$ trials. To verify that the ACC and dlPFC exhibited dissociable patterns of activity, we entered data into an additional ANOVA with "region" as an additional factor. A three-way region (dlPFC, ACC) by switch (switch, stay) by trial type ( $i I, i C, c I, c C$ ) interaction $\left(F_{(3,69)}=3.3 ; p<0.03\right)$ confirmed our observation that the patterns of neural activity observed in these regions were statistically dissociable.

\section{Task-specific cognitive control?}

A closer look at the form of the interaction for current incongruent $(I)$ trials revealed that parameter estimates in the dlPFC were lower on stay-iI trials not only relative to switch-iI $\left(t_{(23)}=5.25 ; p<1 \times\right.$ $10^{-4}$ ) trials, but also relative to both stay- $c I\left(t_{(23)}=2.77 ; p<0.02\right)$ and switch-cI $\left(t_{(23)}=2.3 ; p<0.04\right)$ trials (Fig. $4 C)$. Moreover, activity levels on switch-iI were not significantly greater than those on switch-cI or stay-cI trials (although there was a trend toward dlPFC being more active for switch-iI trials than for switch-cI trials, with $p=0.09$ ). The observed interaction was thus primarily attributable to a relative reduction of activity on stay-iI trials, that is, in which the previous trial was single subject.
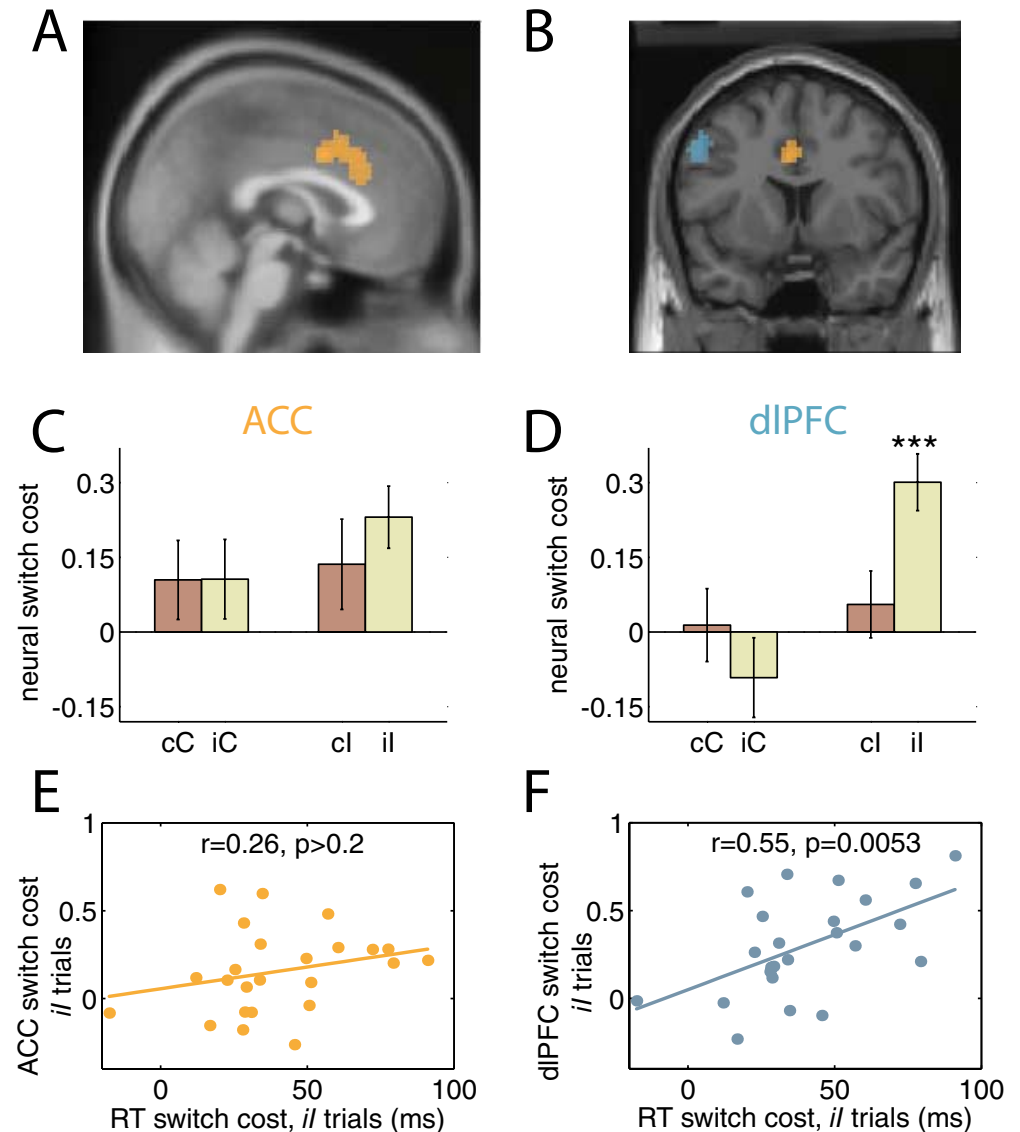

Figure 3. Brain activity in the ACC and dIPFC. $A$, Sagittal $(x=0)$ view of ACC region (left; orange) responding to switch $>$ stay. $\boldsymbol{B}$, Coronal view $(y=3$ ) of dIPFC region (right; cyan) responding to il-switch $>$ il-stay. See Table 1 for other regions activated by these contrasts. $C$, Bar plots of ACC parameter estimates for switch $>$ stay trials in each condition ( $C C, i C, C l$, and il). D, Equivalent bar plots for the dIPFC. The asterisks denote level of statistical significance $\left({ }^{*} p<0.05 ;{ }^{* *} p<0.01 ;{ }^{* * *} p<0.001\right)$. Error bars represent the SEM. $\boldsymbol{E}, \boldsymbol{F}$, Scatter plots with lines of best linear fit show the correlation between neural switch costs on $i /$ trials in the $\mathrm{ACC}(\boldsymbol{E})$ and dIPFC $(\boldsymbol{F})$ and behavioral switch costs on il trials measured from reaction time data. Each dot represents data for a

incongruent and the task did not change. Interestingly, a tightly matched pattern of activity was visible in the bilateral extrastriate visual regions that were identified along with dlPFC in the iI switching contrast (Table 2), most prominently at a cluster on the left fusiform gyrus (coordinates: $-51,-63,-18$ ) (Fig. 4B) (an 
Table 2. Voxels responsive to switch-il $>$ stay-il, at an uncorrected threshold of $p<0.001$

\begin{tabular}{|c|c|c|c|c|c|c|}
\hline \multirow[b]{2}{*}{ Cluster size } & \multirow[b]{2}{*}{$t$ value } & \multicolumn{3}{|c|}{ MNI coordinate } & \multirow[b]{2}{*}{ Brodmann's area (BA) } & \multirow[b]{2}{*}{ Brain region } \\
\hline & & $x$ & $y$ & $z$ & & \\
\hline 62 & 5.48 & -54 & 0 & 36 & $6 / 8 / 9$ & Caudal dIPFC \\
\hline \multirow[t]{3}{*}{66} & 4.83 & -51 & -63 & -18 & $18 / 19 / 37$ & Fusiform gyrus \\
\hline & 4.79 & -45 & -72 & -15 & & \\
\hline & 4.53 & -42 & -78 & -3 & & \\
\hline 9 & 4.03 & 6 & 15 & 48 & $6 / 8 / 32$ & ACC/pre-SMA \\
\hline \multirow[t]{2}{*}{6} & 4.02 & -3 & 9 & 36 & $24 / 32$ & $\mathrm{ACC}$ \\
\hline & 3.67 & 3 & 3 & 33 & & \\
\hline 6 & 3.70 & 30 & -96 & -6 & 18 & Occipital cortex \\
\hline
\end{tabular}

From left to right are the following: cluster size in voxels $(3 \times 3 \times 3 \mathrm{~mm}$ ); peak $t$ value (with $23 \mathrm{df}$ ); $x, y, z$ coordinates of local maximum in the standard space of Montreal Neurological Institute (MNI) template brain; approximate Brodmann's area $(B A)$; and label given to brain region in the text, if mentioned. pre-SMA, Presupplementary motor area.
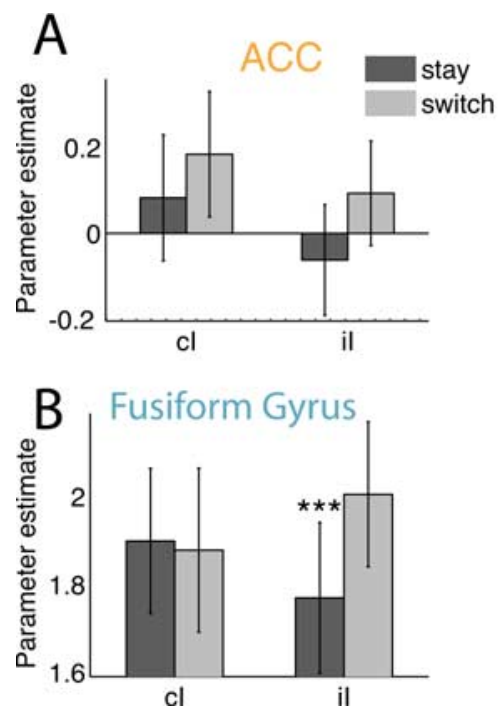

Figure 4. Reduced extrastriate activity on stay-il trials. $\boldsymbol{A}-\boldsymbol{C}$, Parameter estimates obtained within the $\operatorname{ACC}(\boldsymbol{A}), \operatorname{dIPFC}(\boldsymbol{B})$, and fusiform region ( $\boldsymbol{C}$ ) for current incongruent trials. dIPFC and fusiform regions show reliable reductions on stay-il trials. The asterisks denote level of statistical significance $\left({ }^{*} p<0.05 ;{ }^{* *} p<0.01 ;{ }^{* * *} p<0.001\right)$. Error bars represent the SEM. $\boldsymbol{D}$, Fusiform gyrus voxels showing less activation on stay-il trials than other incongruent trial types (blue), and voxels showing reduced functional coupling with the dIPFC on stay-il trial relative to other incongruent trials (red). Overlapping voxels are shown in magenta.
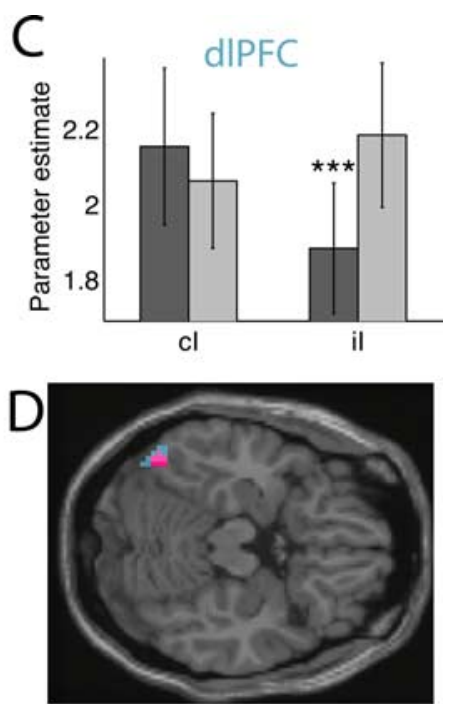

equivalent pattern on the right fusiform gyrus failed to achieve statistical criterion). Within this left fusiform cluster, switch costs were significant on $i$ trials $\left(t_{(23)}=5.6 ; p<1 \times 10^{-5}\right)$ but not other trial types (all values of $p>0.1$ ). Accordingly, greater neural switch costs were observed on $i$ than $c I\left(t_{(23)}=3.6 ; p<0.002\right)$ and $c C\left(t_{(23)}=3.5 ; p<0.002\right)$ trials, and there was a trend for greater switch costs on iI than $i C$ trials $(p<0.09)$, collectively indicating that in the fusiform cortex, too, less fMRI activity was evoked by stay-iI trials than other current incongruent trials.

To test the hypothesis that this pattern of activity in the extrastriate visual cortex was driven by functional coupling with the dlPFC, we tested for psychophysical interactions (PPIs) between the two regions. PPI assesses how the statistical interdependence of the time series in different brain regions varies as a function of experimental condition (Friston et al., 1997). Specifically, we searched across the brain for voxels in which functional coupling with a seed region placed in the dlPFC was reduced on stay-iI relative to other current incongruent trials. Imposing an uncorrected threshold of $p<0.001$, this analysis identified a single cluster of voxels falling just posterior to, but overlapping with, the left fusiform region described above (Fig. 4D). Moreover, when the mean functional coupling with voxels falling directly within the fusiform region was calculated, it was found to be significant $\left(t_{(23)}=2.4 ; p<0.03\right)$, suggesting that less coupling between
dlPFC and the extrastriate visual cortex occurs on stay-iI than other incongruent trials.

Importantly, this pattern of data in the dlPFC and fusiform gyrus differed from that observed in the ACC, in which an analysis restricted to current incongruent trials revealed no interaction between previous trial congruence and switch (Fig. 4A).

\section{Brain-behavior correlations}

We were also interested in how individual differences in behavioral sensitivity predict neural activity associated with task switching. We thus assessed the subjectby-subject correlation between behavioral and neural switch costs on $i I, i C, c I$, and $c C$ trials separately for the ACC and dlPFC. Interestingly, a relationship between neural activity and the RT cost of switching was observed in the dlPFC uniquely on iI trials: in other words, those subjects that were fastest on stay-iI trials compared with switch-iI trials exhibited the greatest reduction in dlPFC activity on stay-iI trials, again compared with switch-iI trials $(r=$ $0.55 ; p<0.006$ ) (Fig. $3 F)$. No such relationship was observed in the ACC (Fig. 3E), nor were reliable correlations obtained for $c I$, $i C$, or $c C$ trials in either region (all absolute $r$ values $<0.4$; all values of $p>0.1$ ) (supplemental Table 2, available at www. jneurosci.org as supplemental material).

\section{Interactions between dIPFC and ACC}

A prominent view argues that the flow of neural information from the ACC to the dlPFC is required for efficient cognitive control (Kerns et al., 2004; Botvinick, 2007). Nevertheless, the empirical support for this view remains somewhat scanty, perhaps in part because of the difficulty in using direction-specific ("causal") connectivity techniques when the input routes are unknown, as is currently the case in the prefrontal cortex. To assess ACC-dlPFC interactions, thus, we took two approaches. First, we placed a PPI seed region in the ACC and searched for voxels in the dlPFC for which coupling was enhanced on switch relative to stay trials. However, no such voxels were identified, nor was the correlation with the mean signal taken from our dlPFC ROI found to be statistically reliable $(p>0.1)$. Second, we adopted a technique widely cited as demonstrating an interdependence between the dlPFC signal and ACC activity when conflict adaptation is occurring (Kerns et al., 2004). This method involves ex- 
tracting the raw fMRI signal in the dlPFC and ACC peaks, and calculating how ACC activity correlates with dlPFC activity on the subsequent trial as a function of condition. Interestingly, although we replicated the finding that dIPFC activity is predicted by ACC activity on trial $n-1$, we found that this was the case not just for $i$ trials, but for all trial types, suggesting a generic coactivation of the medial and lateral PFC rather than a neural correlate of cognitive control. For a detailed description of these analyses, we refer the reader to supplemental Results 1 (available at www. jneurosci.org as supplemental material).

\section{Control analyses}

Finally, to rule out alternative explanations for our results, we conducted a control analysis in which we additionally excluded trials on which any feature of the stimulus ensemble (either word or triangle) was repeated from trial to trial ("partial repetitions"). This analysis served to eliminate any costs associated with overcoming the binding between previously integrated features that may have contributed to our data. Notably, the ACC still exhibited a main effect of switch $\left(F_{(1,23)}=13.2 ; p<0.002\right)$ but no higher interactions (all values of $p>0.5$ ), whereas the dlPFC exhibited a main effect of switch $\left(F_{(1,23)}=8.8 ; p<0.007\right)$, a switch by congruence interaction $\left(F_{(1,23)}=9.6 ; p<0.005\right)$, a switch by previous congruence $\left(F_{(1,23)}=11.0 ; p<0.003\right)$, and a marginally significant three-way interaction $\left(F_{(1,23)}=3 ; p<0.1\right)$. All effects were in the same direction as in the original analysis. The loss of statistical power is most likely attributable to a sharp reduction in trial counts in each condition for this control analysis.

\section{Discussion}

\section{Summary}

The ACC and dIPFC are both engaged when subjects switch between tasks, but the relative contributions of these regions to task switching have yet to be deciphered. Here, we report new evidence that these regions respond in a dissociable manner during task switching, when the dimension of the relevant information alternates between trials. The ACC was activated by task switching mostly regardless of the demands of the current or previous trial, whereas the caudal dlPFC responded to switch cost only for iI trials. We propose that at least two neurocomputational processes underpin task level control (Summerfield and Koechlin, 2009). First, task sets must be assigned a level of priority; in other words, for a given episode, correct outcomes must be assigned to possible actions (for example, specifying that, for task 1, response $P$ is correct given stimulus $Q$ ). This motivational updating occurs whenever a behavioral episode with new rules is initiated but does not depend on the immediate selection demands of the task. We thus associate this process with the ACC (Rushworth et al., 2004). Second, interference must be overcome for this "active" task to be implemented effectively, in the face of competition from rivalrous task sets. This control process, which we have previously termed "contextual control" (Koechlin and Summerfield, 2007), comes into play when the current trial involves conflict between competing task sets (i.e., is incongruent) and the appropriate task set has not been selected yet, and we thus argue that it depends on the caudal dlPFC (Kerns et al., 2004; Brass et al., 2005).

\section{Resolving interference}

Our behavioral analyses confirm previous reports that switch costs depend on whether a rival task set was implemented in a controlled manner on the previous trial (Goschke, 2000; Brown et al., 2007). Behavioral switch costs were enhanced for the second of two incongruent (iI) trials, presumably because of the increased burden of resolving proactive interference from the task set implemented on the previous trial. This finding is reminiscent of the heightened behavioral cost observed when switching away from a more demanding task (Allport, 1994) and supports a literature arguing that overcoming interference from processes associated with the previous task is a major determinant of the cost of task switching (Yeung et al., 2006).

We observed a neural correlate of this effect in the caudal dlPFC. Intriguingly, this effect was driven by a reduction in dlPFC activity for stay-iI trials, those on which the task at hand had already been implemented in a controlled manner on the immediately precedent trial, compared with other incongruent trials (see supplemental Discussion, available at www.jneurosci. org as supplemental material). The dlPFC thus displayed not so much a "switch cost" but rather a "stay benefit," presumably because the control processes required for task implementation were already in place. Some insight into the nature of these control processes is provided by the additional finding that a visual region falling on the fusiform gyrus, as well as functional coupling between this extrastriate visual region and the dlPFC, both exhibited a matching profile of activity, with reductions on stay-iI trials relative to other incongruent trials. Our data thus draw an explicit link between trial-by-trial adjustment in cognitive control and the top-down regulation of activity in task-relevant sensory regions, extending previous findings that posit amplificatory frontoposterior connectivity as the source of adaptation to conflict in the visual cortex (Egner and Hirsch, 2005). These data are highly consistent with prominent views of how the brain faces down competition among competing perceptual codes (Desimone and Duncan, 1995; Kastner et al., 1999; Miller and Cohen, 2001).

\section{Assigning motivational priority}

Importantly, however, this interaction between task switching and previous and current incongruence occurred alongside a global main effect of task switching in reaction times. In other words, statistically significant behavioral switch costs were observed even when $c C, i C$, and $c I$ trials were considered in isolation. On switch- $c C$ and switch- $i C$ trials, in which the current trial was congruent, there was theoretically no need for the subject to implement the switched-to task, because a response made in concert with either the previously active task, or the currently active task, would both yield a correct response. Similarly, on switch-cI trials, proactive interference is greatly reduced, because the previous trial did not demand a choice among conflicting task sets. The finding that switch costs nevertheless occurred on these trials suggests the presence of another time-consuming process underlying the task selection/deselection, which is independent of the demand associated with retrieving the previously implemented task set.

A correlate of this demand-independent switch cost was observed in the ACC, in which a main effect of switch occurred in the absence of any interaction with current or previous trial congruence. We argue that the ACC updates the priority associated with a new task set, regardless of whether that task requires controlled implementation on the immediate trial. This is consistent with the notion that ACC contributes to decision making by assigning outcomes to actions (Rushworth et al., 2004; Walton et al., 2007), thereby allowing resources to be allocated to a given task on the basis of its motivational significance. Moreover, a strong current in the recent neuroimaging literature emphasizes the role the ACC plays in configuring task-related information for a future episode: for example, by responding to instruction cues across a wide variety of tasks and domains (Dosenbach et al., 
2006), particularly when those cues carry clear motivational information (Luks et al., 2002; Brown and Braver, 2005; Hester et al., 2007; Sohn et al., 2007; Aarts et al., 2008).

\section{ACC-dlPFC interactions}

The ACC and dlPFC are often coactive during cognitive tasks, and correspondingly, it has not proved easy to disentangle their relative contributions to executive function. One influential theory argues that, whereas the joint function of the ACC and dlPFC is to overcome sensorimotor conflict, the labor is divided between them such that the ACC monitors for and detects the presence of conflict, whereas the dIPFC engages the top-down processes required to deal with it (Kerns et al., 2004; Botvinick, 2007). The empirical mainstay of this theory is the oft-replicated finding that the ACC responds to the onset of conflict (e.g., on a $c I$ relative to an iI trial, in a single-task paradigm), whereas dlPFC signals correlate with the success of "conflict adaptation," in which the second of two incongruent trials is dealt with more effectively than the first (Kerns et al., 2004; Kerns, 2006).

One important open issue is whether conflict adaptation reflects a general or specific regulation of dlPFC representations. Our results clearly favor the latter view: if conflict adaptation consisted in a general regulation of cognitive resources in the PFC in response to any resource-demanding event (Kerns et al. 2004), then switches after incongruent trials would presumably be dealt with more efficiently, not less efficiently as reported here and previously (Goschke, 2000; Brown et al., 2007). Instead, in line with the pattern of activation in caudal dlPFC, we suggest that conflict adaptation reflects a persistence of engagement of a specific task from trial to trial, such that, when a task set has received extra activation [on a previous incongruent $(i)$ trial], it does not require modulation of activation on a subsequent incongruent trial unless a switch occurs. This finding draws support from previous observations that conflict adaptation transfers poorly between tasks with different visuospatial properties (Corballis and Gratton, 2003; Mayr et al., 2003).

Second, an oft-cited basis for the conflict detection/implementation hypothesis is the finding that, where control adjustments are required (i.e., on $i$ trials), ACC activity predicts dlPFC activity on the subsequent trial (Kerns et al., 2004; Kerns, 2006). We replicate this finding but found that it occurred indiscriminately (i.e., was also present for $c C, i C$, and $c I$ trials including both switch and stay variants) raising the possibility that it reflects a general (e.g., physiological) correlation in ACC-dlPFC activity, unrelated to cognitive control (unfortunately, previous studies did not report the correlation values for $i C, c I$, or $c C$ trials, precluding an assessment of the specificity of their finding). Although it is highly plausible that the ACC and dIPFC exchange information during executive control, other techniques may be required to characterize this neural interrelationship.

Finally, a taxonomy of the cognitive processes underlying task switching remains a topic of ongoing and often controversial debate (Monsell and Driver, 2000). Although many researchers believe that the time required to configure a new task set accounts for a major portion of the behavioral switch cost, even independent of the priming, retrieval, and interference effects that potentially spill over from the previously active task (Rubinstein et al., 2001; Monsell, 2003; Monsell and Mizon, 2006), others disagree (Logan and Bundesen, 2003; Altmann, 2006). Although our experiment was not designed to speak directly to this debate, the presence of a switch cost that is immune to levels of demand on the previous task set (such that we observed in the ACC) is easier to reconcile with a time-consuming process that configures the priorities associated with a new task set than with one that suppresses interference from a past task. Our data thus offer support for the emerging view that task switching involves at least two distinct neural mechanisms, one that overcomes interference and another that organizes priorities between competing task sets (Crone et al., 2006; Johnston et al., 2007).

\section{References}

Aarts E, Roelofs A, van Turennout M (2008) Anticipatory activity in anterior cingulate cortex can be independent of conflict and error likelihood. J Neurosci 28:4671-4678.

Allport A (1994) Shifting attentional set: exploring the dynamic control of tasks. In: Attention and performance XV (Umilta C, Moscovitch M, eds), pp 421-452. Cambridge, MA: MIT.

Altmann EM (2006) Task switching is not cue switching. Psychon Bull Rev 13:1016-1022.

Aron AR, Monsell S, Sahakian BJ, Robbins TW (2004) A componential analysis of task-switching deficits associated with lesions of left and right frontal cortex. Brain 127:1561-1573.

Botvinick MM (2007) Conflict monitoring and decision making: reconciling two perspectives on anterior cingulate function. Cogn Affect Behav Neurosci 7:356-366.

Botvinick MM, Braver TS, Barch DM, Carter CS, Cohen JD (2001) Conflict monitoring and cognitive control. Psychol Rev 108:624-652.

Brainard DH (1997) The psychophysics toolbox. Spat Vis 10:433-436.

Brass M, Derrfuss J, Forstmann B, von Cramon DY (2005) The role of the inferior frontal junction area in cognitive control. Trends Cogn Sci 9:314-316.

Brown JW, Braver TS (2005) Learned predictions of error likelihood in the anterior cingulate cortex. Science 307:1118-1121.

Brown JW, Reynolds JR, Braver TS (2007) A computational model of fractionated conflict-control mechanisms in task-switching. Cognit Psychol 55:37-85

Carter CS, Braver TS, Barch DM, Botvinick MM, Noll D, Cohen JD (1998) Anterior cingulate cortex, error detection, and the online monitoring of performance. Science 280:747-749.

Corballis PM, Gratton G (2003) Independent control of processing strategies for different locations in the visual field. Biol Psychol 64:191-209.

Crone EA, Wendelken C, Donohue SE, Bunge SA (2006) Neural evidence for dissociable components of task-switching. Cereb Cortex 16:475-486.

Desimone R, Duncan J (1995) Neural mechanisms of selective visual attention. Annu Rev Neurosci 18:193-222.

Dosenbach NU, Visscher KM, Palmer ED, Miezin FM, Wenger KK, Kang HC, Burgund ED, Grimes AL, Schlaggar BL, Petersen SE (2006) A core system for the implementation of task sets. Neuron 50:799-812.

Egner T, Hirsch J (2005) Cognitive control mechanisms resolve conflict through cortical amplification of task-relevant information. Nat Neurosci 8:1784-1790

Friston KJ, Buechel C, Fink GR, Morris J, Rolls E, Dolan RJ (1997) Psychophysiological and modulatory interactions in neuroimaging. Neuroimage $6: 218-229$

Goschke T (2000) Intentional reconfiguration and involuntary persistence in task-set switching. In: Attention and performance XVIII (Monsell S, Driver J, eds), pp 331-355. Cambridge, MA: MIT.

Gratton G, Coles MG, Donchin E (1992) Optimizing the use of information: strategic control of activation of responses. J Exp Psychol Gen 121:480-506

Hester R, Barre N, Mattingley JB, Foxe JJ, Garavan H (2007) Avoiding another mistake: error and posterror neural activity associated with adaptive posterror behavior change. Cogn Affect Behav Neurosci 7:317-326.

Hommel B, Proctor RW, Vu KP (2004) A feature-integration account of sequential effects in the Simon task. Psychol Res 68:1-17.

Jersild AT (1927) Mental set and shift. Arch Psychol 89:81.

Johnston K, Levin HM, Koval MJ, Everling S (2007) Top-down controlsignal dynamics in anterior cingulate and prefrontal cortex neurons following task switching. Neuron 53:453-462.

Kastner S, Pinsk MA, De Weerd P, Desimone R, Ungerleider LG (1999) Increased activity in human visual cortex during directed attention in the absence of visual stimulation. Neuron 22:751-761.

Kerns JG (2006) Anterior cingulate and prefrontal cortex activity in an FMRI study of trial-to-trial adjustments on the Simon task. Neuroimage $33: 399-405$. 
Kerns JG, Cohen JD, MacDonald AW 3rd, Cho RY, Stenger VA, Carter CS (2004) Anterior cingulate conflict monitoring and adjustments in control. Science 303:1023-1026.

Koechlin E, Summerfield C (2007) An information theoretical approach to prefrontal executive function. Trends Cogn Sci 11:229-235.

Logan GD, Bundesen C (2003) Clever homunculus: is there an endogenous act of control in the explicit task-cuing procedure? J Exp Psychol Hum Percept Perform 29:575-599.

Luks TL, Simpson GV, Feiwell RJ, Miller WL (2002) Evidence for anterior cingulate cortex involvement in monitoring preparatory attentional set. Neuroimage 17:792-802.

Mayr U, Awh E, Laurey P (2003) Conflict adaptation effects in the absence of executive control. Nat Neurosci 6:450-452.

Miller EK, Cohen JD (2001) An integrative theory of prefrontal cortex function. Annu Rev Neurosci 24:167-202.

Monsell S (2003) Task switching. Trends Cogn Sci 7:134-140.

Monsell S, Driver J (2000) The control of cognitive processes: attention and performance XVIII. Cambridge, MA: MIT.

Monsell S, Mizon GA (2006) Can the task-cuing paradigm measure an endogenous task-set reconfiguration process? J Exp Psychol Hum Percept Perform 32:493-516.
Monsell S, Sumner P, Waters H (2003) Task-set reconfiguration with predictable and unpredictable task switches. Mem Cognit 31:327-342.

Rogers RD, Monsell S (1995) The costs of a predictable switch between simple cognitive tasks. J Exp Psychol Gen 124:207-231.

Rubinstein JS, Meyer DE, Evans JE (2001) Executive control of cognitive processes in task switching. J Exp Psychol Hum Percept Perform 27:763-797.

Rushworth MF, Walton ME, Kennerley SW, Bannerman DM (2004) Action sets and decisions in the medial frontal cortex. Trends Cogn Sci 8:410-417.

Sohn MH, Albert MV, Jung K, Carter CS, Anderson JR (2007) Anticipation of conflict monitoring in the anterior cingulate cortex and the prefrontal cortex. Proc Natl Acad Sci U S A 104:10330-10334.

Summerfield C, Koechlin E (2009) Decision-making and prefrontal executive function. In: The cognitive neurosciences IV (Gazzaniga MS, ed). Cambridge, MA: MIT, in press.

Ullsperger M, Bylsma LM, Botvinick MM (2005) The conflict adaptation effect: it's not just priming. Cogn Affect Behav Neurosci 5:467-472.

Walton ME, Croxson PL, Behrens TE, Kennerley SW, Rushworth MF (2007) Adaptive decision making and value in the anterior cingulate cortex. Neuroimage 36 [Suppl 2]:T142-T154.

Yeung N, Nystrom LE, Aronson JA, Cohen JD (2006) Between-task competition and cognitive control in task switching. J Neurosci 26:1429-1438. 